\title{
Sedentarismo y salud: efectos beneficiosos de la actividad física en estudiantes universitarios
}

\author{
María Belén Ramírez Prieto. Universidad de Granada \\ Manuel Raya Franco. Universidad de Granada \\ Marta Ruiz del Rio. Universidad de Granada
}

Recepción: 26.02.2018| Aceptado: 5.03.2018

Correspondencia a través de ORCID: María Belén Ramírez

iD $0000-0003-4212-8574$

Citar: Ramirez, MB., Raya, M. y Ruiz, M. (2018). Sedentarismo y salud: efectos beneficiosos de la actividad física en estudiantes universitarios. ReiDoCrea, 7, 79-84.

\begin{abstract}
Resumen: El abundante análisis epidemiológico que se lleva haciendo desde unas décadas atrás sobre la actividad física, ha alcanzado conclusiones muy uniformes acerca de los beneficios, tanto físicos como psicológicos, de su práctica regular. Objetivos/Hipótesis: Contrastar si los estudiantes universitarios físicamente activos tendrían un mejor autoconcepto, mayor bienestar subjetivo, así como, un menor estrés percibido. Método: Se utilizó una muestra de 30 sujetos universitarios y se llevó a cabo la administración de diferentes escalas. Los datos obtenidos fueron analizados con una prueba T- Student para muestras independientes. Resultados: Los datos obtenidos confirmaron que los estudiantes universitarios activos tenían un mejor autoconcepto, mayor bienestar subjetivo y un menor estrés percibido. Conclusiones: La actividad física trae consigo numerosos beneficios a nivel psicológico, el conocimiento de los mismos podría implicar una mayor participación e implicación de la población universitaria.
\end{abstract}

Palabras clave: Estudiantes | Deporte

Sedentary Lifestyle and Health: BeneficialEeffects of Physical Activity in University Students

\begin{abstract}
The abundant epidemiological analysis that has been carried out during the last decades about physical activity has reached very uniform conclusions about the psychological and physical benefits of the regular practice of physical activity. Objective/Hypothesis: to contrast if physically active university students would have a better self-concept and greater subjective well-being as well as less perceived stress. Method: a sample of 30 university subjects was used and different scales were administered. The information obtained was analyzed by Student's T- test for independent samples. Results: The information obtained confirmed that active university students had a better self-concept, greater subjective well-being and less perceived stress. Conclusions: physical activity brings numerous psychological benefits and knowledge of these may imply greater participation and involvement of the university population.
\end{abstract}

Keywords: Students | Sports

\section{Introducción}

En las dos últimas décadas se han llevado a cabo numerosos estudios que demuestran los beneficios producidos en la salud debido a la práctica de actividad física regular (Álvarez, Cuevas, Lara y González, 2015). Algunos de los cuales han sido demostrados y que veremos en este estudio, están relacionados con aquellas personas que tienen estilos de vida saludables y presentan, a su vez, un mayor bienestar psicológico (García, Vieira y Rodrigues, 2014). Estas personas se perciben como más sanas, con menor estrés y presentan mejor estado de ánimo que aquellas otras que no realizan ningún tipo de actividad física (Jiménez, Martínez, Miró y Sánchez, 2008). Otros beneficios asociados son una mayor satisfacción con respecto a su cuerpo, amor propio, así como, la presencia de mejores autopercepciones físicas (Tremblay, Colley, Saunders, Healy y Owen, 2010). Asimismo y relacionado con lo anterior, estudios llevados a cabo por Esnaola y Revuelta (2009) han encontrado correlaciones significativas entre aquellas personas que se perciben bien en el 
autoconcepto general, el autoconcepto físico y los subdominios específicos, teniendo un valor percibido de la actividad física alta, así como una expectativa o rendimiento esperado positivo y perciben con menor dificultad la actividad física.

Otro de los beneficios para incrementar el ritmo de actividad en el individuo, ha sido la constatación de que el ejercicio físico actúa como medio preventivo contra el estrés ambiental en estudiantes. Esto fue observado en la investigación de Holmes (1993): los sujetos con mejor forma física eran capaces de soportar eventos vitales estresantes mejor que los de menor forma física, sin desarrollar procesos depresivos. En esta línea, VanKim y Nelson (2013) encontraron que el ejercicio físico moderaba la relación entre el estrés ambiental y la depresión. Concluyendo que aquellos estudiantes universitarios que lleven a cabo una vida física activa, tendrán una mejor salud mental y percepción del estrés. Por lo tanto, los hábitos de salud de estudiantes universitarios suponen una preocupación especial, ya que ellos representan una gran parte de la población juvenil y están en una etapa de sus vidas durante la cual se producen importantes modificaciones en el estilo de vida (Varela, Duarte, Salazar, Lema y Tamayo, 2011).

Una de las preguntas que pueden surgirnos es, ¿cuándo hablamos de sedentarismo? Según Varela et al. (2011) el sedentarismo hace referencia a la falta de actividad física en la que el individuo en cuestión no realiza una cantidad mínima de movimiento diario, por lo menos entre 25 y 30 minutos.

En el terreno universitario un estudio realizado por Martínez, Puig y García (2014) indica que a pesar de haber campus universitarios donde se ofertan oportunidades para la realización de actividad física, esto parece no ser suficiente. El problema reside en la falta de información acerca de las barreras manifestadas por la población universitaria española. Las principales conclusiones llevadas a cabo, indican bajas puntuaciones en el número total de barreras. Sin embargo, se identificaron puntuaciones más altas en barreras externas como "demasiado trabajo" o "falta de tiempo" que internas. No obstante, hay otros argumentos internos empleados, aunque en menor medida, tal como "hoy estoy cansado", que constituyen otra de las vías de escape. Por ello, se considera importante hacer hincapié en los aspectos positivos del ejercicio físico para disipar o reducir estos factores asociados, tanto externos como internos (Gómez, Gallegos y Extremera, 2010).

Otro dato constatado ha sido el proporcionado por Jiménez et al. (2008) en el que se demuestra que el tipo de ejercicio practicado parece no tener influencia sobre las distintas variables dependientes consideradas (salud percibida, estrés y estado de ánimo). Tanto la actividad física aeróbica como el entrenamiento en resistencia contribuyen a mejorar el bienestar psicológico de las personas. Posiblemente, más que el tipo de ejercicio practicado, lo relevante es el tiempo semanal dedicado a la práctica de actividad física.

Por todo lo comentado anteriormente, este estudio pretende confirmar si la actividad física provoca un mayor bienestar psicológico, autoconcepto sobre uno mismo y una disminución del estrés percibido en estudiantes universitarios.

\section{Objetivos}

Este estudio tiene como hipótesis primaria confirmar si la actividad física va asociada a efectos beneficiosos en la población universitaria físicamente activa. Dentro este objetivo general, se plantean los siguientes: 
1. Si la actividad física provoca un mayor bienestar psicológico en estudiantes universitarios.

2. Si la actividad física va asociada a un mejor autoconcepto en estudiantes universitarios.

3. Si la actividad física va a asociada a una menor percepción del estrés en estudiantes universitarios.

\section{Métodos}

\section{Participantes}

En el presente estudio participaron voluntariamente 30 personas de nacionalidad española que estaban realizando estudios universitarios con edades comprendidas entre los 18 y los 25 años, siendo la media de las edades de 21,1 (DT = 2,123). La distribución del género de la muestra fue del $50 \%$, es decir, la mitad eran hombres y la otra mitad mujeres. Con respecto a la actividad física, los participantes fueron clasificados como sedentarios o como personas activas, encontrándose en ambos géneros la mitad de participantes que presentaban un nivel de actividad $u$ otro.

\section{Materiales}

En primer lugar, para introducir a los participantes en el estudio de investigación se les suministró un cuestionario empleado en el estudio llevado a cabo por Jiménez et al. (2008) para obtener información sobre el grado de salud percibida la cual va de 1 (muy mala) a 5 (excelente), así como, preguntas relacionadas con la práctica de ejercicio físico (frecuencia de la práctica de ejercicio y tiempo que llevan practicando ese deporte).

En segundo lugar, se administró la Escala de Estrés Percibido (Perceived Stress Scale, PSS) elaborada por Cohen, Kamarck y Mermelstein (1983) en su versión española adaptada por Remor (2001). Esta escala es un instrumento de autoinforme que evalúa el nivel de estrés percibido durante el último mes. Consta de 14 ítems con un formato de respuesta de una escala de cinco puntos que va desde 0 (nunca) hasta 4 (muy a menudo). El alfa de Cronbach de la escala fue de 0,894.

En tercer lugar, se aplicó el Autoconcepto Forma 5 (AF-5) de García y Musitu (2001), un cuestionario compuesto por 30 ítems que evalúan el autoconcepto de la persona evaluada en sus vertientes Social, Académica/Profesional, Emocional, Familiar y Física. Se definieron 6 ítems de cada una de las cinco dimensiones. El formato de respuesta va de 1 a 99, representando una puntuación baja un nivel alto de desacuerdo con la frase, y una puntuación alta lo contrario. El alfa de Cronbach de la escala fue de 0,906.

Por último, se hizo uso de la Escalas de Bienestar Psicológico de Ryff (Scales of pshychological wellbeing-reduced) revisada por Díaz et al. (2006) que sirve para evaluar el bienestar psicológico de una persona en base a seis dimensiones: dominio del entorno, crecimiento personal, propósito en la vida, autonomía, autoaceptación y relaciones positivas con otros. Consta de 29 ítems con un formato de respuesta tipo Likert de seis puntos ( $1=$ totalmente en desacuerdo y $6=$ totalmente de acuerdo). El alfa de Cronbach de la escala fue de 0,932. 


\section{Procedimiento}

Los instrumentos utilizados en la investigación fueron presentados a los participantes en un documento único. La colaboración de los participantes fue totalmente anónima y voluntaria, insistiendo en la sinceridad de las respuestas. Por otro lado, no recibieron ninguna compensación económica ni académica por su participación en el estudio. El ambiente donde se llevó a cabo la administración fue calmado y tranquilo para favorecer la relajación y concentración de los mismos. Durante la aplicación de los cuestionarios, cada participante contaba con la presencia de uno de los investigadores con el fin de solventar toda duda que pudiera surgir en la comprensión, tanto de los ítems que componen los distintos instrumentos como de las instrucciones establecidas al inicio de la administración. Cada aplicación duró aproximadamente 40 minutos.

\section{Resultados}

Se procedió a constatar si existían diferencias significativas entre deportistas y personas sedentarias en cuanto al estrés percibido, autoconcepto y bienestar subjetivo, así como, en las diferentes subescalas pertenecientes a estos dos últimos test proporcionados. Para ello, se procedió a realizar el análisis de datos con una prueba t de student para muestras independientes.

En primer lugar y con respecto al autoconcepto general, los datos muestran diferencias significativas entre personas activas físicamente y sedentarias en la con $t(28)=4,95, p<.001$. Por lo que se interpreta que los deportistas tienen un mejor autoconcepto general. Además, las diferentes subescalas pertenecientes a dicho test, muestran diferencias significativas, tales son: autoconcepto académico con $t(28)=$ $2,171, p=.039$; autoconcepto social con $t(28)=2,617, p=.014$; autoconcepto emocional con $t(28)=2,736, p=.011$ y la relativa al autoconcepto físico con $t(28)=6,339, p=$ $<.001$. No obstante, la escala referida al autoconcepto familiar no resultó significativa con $t(28)=1,25, p=.222$.

Gráfico 1. Medias totales en autoconcepto en función de la actividad física

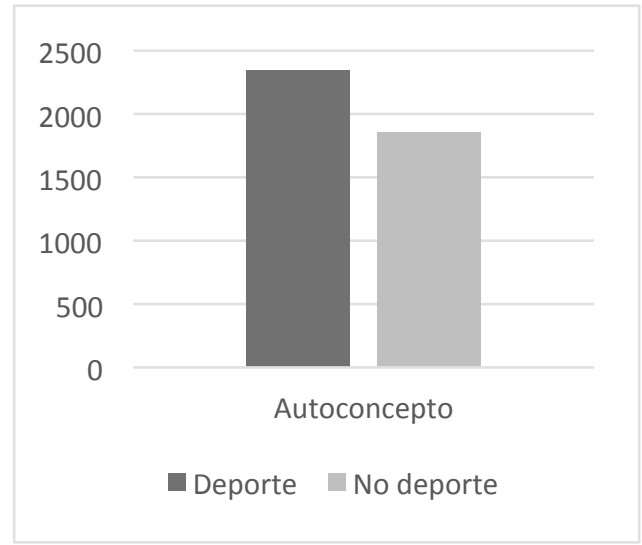

En segundo lugar y en cuanto al bienestar subjetivo, los datos mostraron diferencias significativas con $t(28)=2,99, p=.006$. Además diferentes subescalas pertenecientes a esta variable también muestran valores significativos, tales son: autoaceptación con $t(28)=2,734, p=.011$; autonomía con $t(28)=2,835, p=.008$; dominio del entorno con $t(28)=2,456, p=.021$; propósito en la vida con $t(28)=3,384, p=.002$. Sin embargo, dos subescalas no presentan diferencias significativas, tales son: relaciones positivas con $t(28)=.686, p=.499$ y crecimiento personal con $t(28)=1,905, p=.067$. 
Gráfico 2. Medias totales en bienestar subjetivo en función de la actividad física

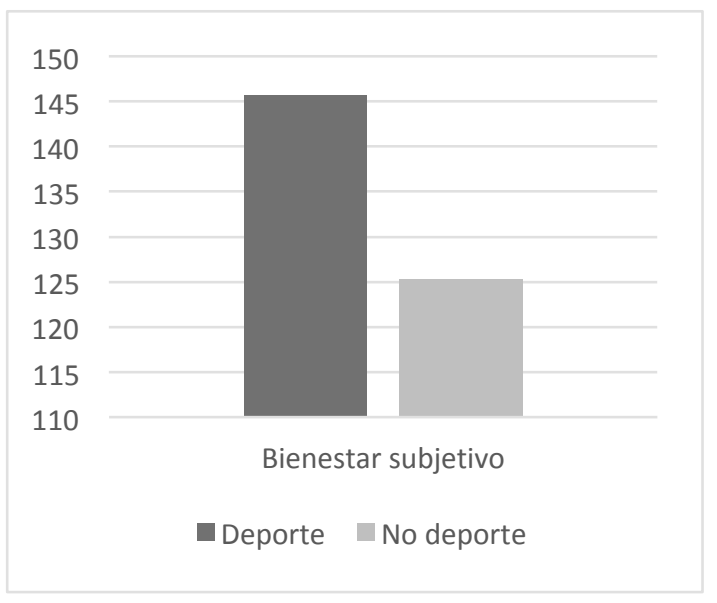

Por último, fueron halladas diferencias significativas en cuanto al estrés percibido con $t(28)=2,004, p=.05$.

Gráfico 3. Medias totales en estrés percibido en función de la actividad física

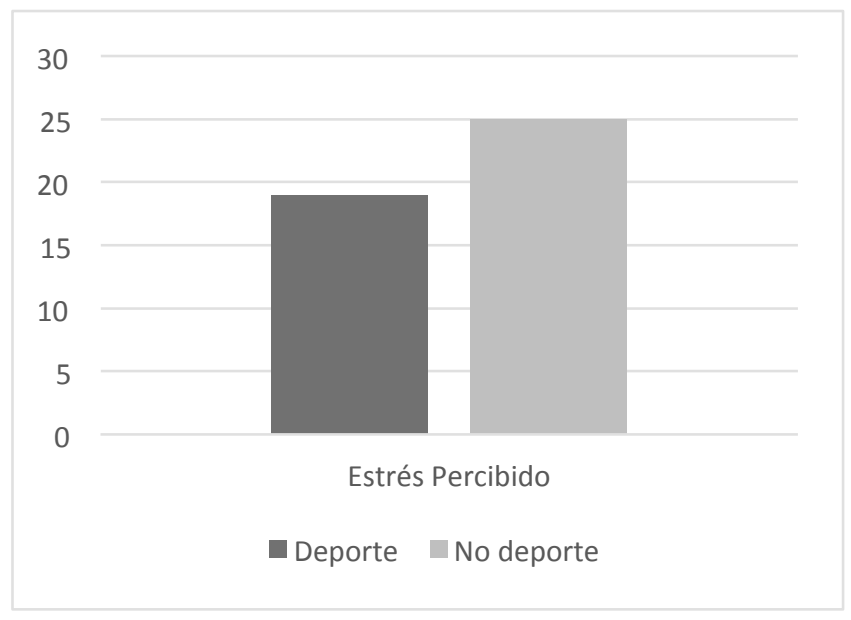

\section{Discusión}

En el presente estudio se quiso comprobar si la actividad física provocaba un mayor bienestar psicológico, un mejor autoconcepto y una disminución del estrés en estudiantes universitarios. Según Tremblay et al. (2010) algunos de los beneficios asociados a la práctica deportiva estaban relacionados con respecto a su cuerpo, amor propio, así como, mejores autopercepciones físicas. Siendo esto confirmado por los resultados de esta investigación, en la que se muestra cómo la práctica deportiva se relaciona con un mayor autoconcepto social, académico, emocional y físico, por lo que se puede confirmar que los universitarios activos físicamente se perciben a sí mismos de manera más positiva.

Por otro lado y, según VanKim y Nelson (2013): aquellos estudiantes universitarios que llevaran a cabo una vida física activa, tendrán una mejor salud mental y percepción del estrés. Tal y como se puede apreciar en las escalas correspondientes al estrés 
percibido se vieron diferencias significativas y en cuanto al bienestar psicológico, se corroboró la hipótesis planteada por estos autores. A partir de estos resultados, se deduce que la práctica deportiva suscita bajos niveles de estrés y un mayor bienestar psicológico.

En cuanto a las limitaciones de este estudio, cabe señalar que la muestra evaluada podría no ser representativa, ya que se cuenta únicamente con 30 sujetos. Se sugiere la realización de futuras investigaciones que superen esta limitación metodológica. Así mismo, el muestreo debería ser más objetivo y aleatorio (probabilístico) ya que podrían darse sesgos debido a la manipulación para la obtención intencionada de algunos resultados. Además, se podría haber clasificado a los deportistas en función del tipo de actividad física realizada ya que todos los sujetos no practicaban el mismo deporte ni le dedicaban el mismo tiempo.

Por último, resaltar que este artículo puede ser de gran interés para estudiantes universitarios e incluso para las propias universidades. De alguna forma, este trabajo puede servir de impulso para modificar el valor que muchos estudiantes atribuyen a la actividad física. Como consecuencia de toda esta serie de beneficios podría adquirir el ejercicio físico un papel más relevante en sus vidas y lograr una mayor implicación y compromiso de estos, de ahí la importancia de este estudio (su aplicabilidad). Además, las propias universidades podrían verse beneficiadas de todo ello. Según Martínez, Puig y García, (2014) hay numerosos campus universitarios que ofertan oportunidades deportivas y, sin embargo, no parece ser suficiente para realización de actividad física. Si se lograra una mayor motivación tras conocerse y fomentarse los efectos beneficiosos de la actividad deportiva, aumentaría la participación en estos campus y se haría uso de las instalaciones mostrando una mayor funcionalidad de estas.

\section{Referencias}

Álvarez Rivera, L., Cuevas Ferrera, R., Lara Pot, A. y González Hernández, J. (2015). Diferencias del autoconcepto físico en practicantes y no practicantes de actividad física en estudiantes universitarios. Cuadernos De Psicología Del Deporte, 15(2), 27-34

Esnaola, I. y Revuelta, L. (2009). Relaciones entre la actividad física, autoconcepto físico, expectativas, valor percibido y dificultad percibida. Acción Psicológica, 6(2), 45-52.

García, A., Vieira, L., \& Rodrigues, N. (2014). Could Physical Activity Improve Healthy Lifestyles and Psychological WellBeing?. Advances In Social Sciences Research, 1(3), 106117.

Gómez-López, M., Gallegos A., \& Extremera A. (2010). Perceived barriers by university students in the practice of physical activities. Journal of Sports Science and Medicine, 9(3), 37481.

Jiménez, M., Martínez, P., Miró, E. y Sánchez, A. (2008). Bienestar psicológico y hábitos saludables: ¿están asociados a la práctica de ejercicio físico? International Journal of Clinical and Health Psychology, 8(1), 185-202.
Palenzuela, D., Gutiérrez Calvo, M. y Avero, P. (1998). Ejercicio físico regular como un mecanismo de protección contra la depresión en jóvenes. Psicothema, 10,(1), 29-39.

Tremblay, M., Colley, R., Saunders, T., Healy, G., \& Owen, N. (2010). Physiological and health implications of a sedentary lifestyle. Applied Physiology, Nutrition, And Metabolism, 35(6), 725-740.

Valera, M., Duarte, C., Salazar, I., Lema, L. y Tamayo, J. (2011). Actividad física y sedentarismo en jóvenes universitarios de Colombia: prácticas, motivos y recursos para realizarlas. Colombia Médica, 42 (3), 269-277.

VanKim, N., \& Nelson, T. (2013). Vigorous Physical Activity, Mental Health, Perceived Stress, and Socializing among College Students. American Journal Of Health Promotion, 28(1), 7-15.

Varela-Mato, V., Cancela, J., Ayan, C., Martín, V., \& Molina, A. (2012). Lifestyle and Health among Spanish University Students: Differences by Gender and Academic Discipline. International Journal Of Environmental Research And Public Health, 9(12), 2728-2741. 